\title{
Longest paths in random Apollonian networks and largest $r$-ary subtrees of random $d$-ary recursive trees
}

\author{
Andrea Collevecchio* \\ School of Mathematical Sciences, Monash University, and \\ Ca' Foscari University, Venice \\ andrea.collevecchio@monash.edu \\ Abbas Mehrabian \\ Department of Combinatorics and Optimization, University of Waterloo \\ amehrabi@uwaterloo.ca \\ Nick Wormald ${ }^{\dagger}$ \\ School of Mathematical Sciences, Monash University \\ nick. wormald@monash.edu
}

\begin{abstract}
Let $r$ and $d$ be positive integers with $r<d$. Consider a random $d$-ary tree constructed as follows. Start with a single vertex, and in each time-step choose a uniformly random leaf and give it $d$ newly created offspring. Let $\mathcal{T}_{t}$ be the tree produced after $t$ steps. We show that there exists a fixed $\delta<1$ depending on $d$ and $r$ such that almost surely for all large $t$, every $r$-ary subtree of $\mathcal{T}_{t}$ has less than $t^{\delta}$ vertices.

The proof involves analysis that also yields a related result. Consider the following iterative construction of a random planar triangulation. Start with a triangle embedded in the plane. In each step, choose a bounded face uniformly at random, add a vertex inside that face and join it to the vertices of the face. In this way, one face is destroyed and three new faces are created. After $t$ steps, we obtain a random triangulated plane graph with $t+3$ vertices, which is called a random Apollonian network. We prove that there exists a fixed $\delta<1$, such that eventually every path in this graph has length less than $t^{\delta}$, which verifies a conjecture of Cooper and Frieze.
\end{abstract}

AMS 2010 subject classification: 05C80 (60C05, 05C05)

Keywords: random Apollonian networks, random recursive $d$-ary trees, longest paths, Eggenberger-Pólya urns.

*Supported by ARC Discovery Project grant DP140100559.

${ }^{\dagger}$ Supported by Australian Laureate Fellowships grant FL120100125. 


\section{Introduction}

In this paper we study two important random graph models. The first one is a so-called random $d$-ary recursive tree, defined as follows. Let $d>1$ be a positive integer. Consider a random $d$-ary tree evolving as follows. At time 0 it consists of exactly one vertex, $\varrho$. In the first step $\varrho$ gives birth to $d$ offspring. In each subsequent step we pick, uniformly at random, a vertex with no offspring and connect it with exactly $d$ offspring. At time $t$ this random tree is denoted by $\mathcal{T}_{t}$. See Drmota [3] for more on random $d$-ary recursive trees. Let $r$ be a fixed positive integer smaller than $d$ and let $S_{t}$ denote the size of the largest (possibly non-unique) $r$-ary subtree of $\mathcal{T}_{t}$.

We say that a sequence of events $\left\{A_{k}, k \in \mathbb{N}\right\}$ occurs eventually (for large $k$ ) if there exists an almost surely (a.s.) finite random variable $N$ such that $A_{k}$ occurs for all $k \geq N$. In this paper all logarithms are natural.

Theorem 1.1. There exists a fixed $\delta<1$ such that $S_{t}<t^{\delta}$ eventually.

In Section 5.1 we show we can take

$$
\delta=1-\frac{d-r}{e d^{2 d} \log (11 d \log d)}
$$

in this theorem.

The second object we study is a popular random graph model for generating planar graphs with power law properties, which is defined as follows. Start with a triangle embedded in the plane. At each step, choose a bounded face uniformly at random, add a vertex inside that face and join it to the vertices on the face. In this way, one face is destroyed and three new faces are created. We call this operation subdividing the face. After $t$ steps, we have a (random) triangulated plane graph $\mathrm{RAN}_{t}$ with $t+3$ vertices, $3 t+3$ edges, $2 t+1$ bounded faces, and 1 unbounded face. The random graph $\mathrm{RAN}_{t}$ is called a random Apollonian network.

Random Apollonian networks were defined by Zhou, Yan, and Wang [12] (see Zhang, Comellas, Fertin, and Rong [11] for a generalization to higher dimensions), where it was proved that the diameter of $\mathrm{RAN}_{t}$ is probabilistically bounded above by a constant times $\log t$. It was shown in $[12,9]$ that $\mathrm{RAN}_{t}$ exhibits a power law degree distribution for large $t$. The average distance between two vertices in a typical $\mathrm{RAN}_{t}$ was shown to be $\Theta(\log t)$ by Albenque and Marckert [1], and a central limit theorem was proved by Kolossváry, Komjáty, and Vágó [6]. The degree distribution, $k$ largest degrees, $k$ largest eigenvalues (for fixed $k$ ), and diameter were studied by Frieze and Tsourakakis [5]. The asymptotic value of the diameter of a typical $\mathrm{RAN}_{t}$ was determined in [4]. We continue this line of research by studying the asymptotic properties of the longest (simple) paths in $\mathrm{RAN}_{t}$.

Let $\mathcal{L}_{t}$ be a random variable denoting the number of vertices in a longest path in $\mathrm{RAN}_{t}$. All the limits in this paragraph are as $t \rightarrow \infty$. Frieze and Tsourakakis [5] conjectured there exists a fixed $\delta>0$ such that $\mathbb{P}\left(\delta t \leq \mathcal{L}_{t}<t\right) \rightarrow 1$. Ebrahimzadeh, Farczadi, Gao, Mehrabian, Sato, Wormald, and Zung [4] refuted this conjecture and showed there exists a 
fixed $\delta>0$ such that $\mathbb{P}\left(\mathcal{L}_{t}<t /(\log t)^{\delta}\right) \rightarrow 1$. Cooper and Frieze [2] improved this result by showing that for every constant $c<2 / 3$, we have $\mathbb{P}\left(\mathcal{L}_{t} \leq t \exp \left(-\log ^{c} t\right)\right) \rightarrow 1$, and conjectured there exists a fixed $\delta<1$ such that $\mathbb{P}\left(\mathcal{L}_{t} \leq t^{\delta}\right) \rightarrow 1$. The second main result of this paper is the following, which in particular confirms this conjecture.

Theorem 1.2. There exists a fixed $\delta<1$ such that $\mathcal{L}_{t}<t^{\delta}$ eventually.

We can take $\delta=1-4 \times 10^{-8}$, as shown in Section 5.2.

Regarding lower bounds, it was proved in [4] that $\mathcal{L}_{t}>(2 t+1)^{\log 2 / \log 3}$ deterministically, and that $\mathbf{E}\left[\mathcal{L}_{t}\right]=\Omega\left(t^{0.88}\right)$.

We prove the two main theorems by studying a third object, an infinite tree with weighted vertices, which is introduced in Section 2. Then we prove Theorems 1.1 and 1.2 in Sections 3 and 4 , respectively. Note that both of these theorems are existential. In Section 5 we give explicit bounds for the values of $\delta$ in these theorems.

\section{Subtrees of an infinite $d$-ary tree}

Fix positive integers $r, d$ with $r<d$. Let $\mathcal{T}$ be an infinite rooted $d$-ary tree. Denote the root by $\varrho$. We denote by $[\nu, \mu]$ the vertices in the path connecting $\nu$ to $\mu$, including these two vertices. For a vertex $\nu$, denote its distance from $\varrho$ by $|\nu|$, and its offspring by $\nu i$, with $i \in\{1,2, \ldots, d\}$. For $\nu \neq \varrho$, denote by $\nu^{-}$the parent of $\nu$, i.e. the neighbour $\mu$ of $\nu$ with $|\mu|=|\nu|-1$.

To each vertex $\nu$ assign a random variable $X_{\nu} \in(0,1]$, satisfying the following properties. We have $X_{\varrho}=1$. The random variables $X_{\nu}$, for $\nu \in V(\mathcal{T}) \backslash\{\varrho\}$, are identically distributed. Moreover we assume that the vectors $\left(X_{\nu 1}, X_{\nu 2}, \ldots, X_{\nu d}\right)$ are identically distributed and independent, and that $\sum_{i=1}^{d} X_{\nu i}=1$. For any vertex $\nu$, define the random variable

$$
\Upsilon_{\nu}=\min \left\{X_{\nu i_{1}}+X_{\nu i_{2}}+\ldots+X_{\nu i_{d-r}}: 1 \leq i_{1}<i_{2}<\ldots<i_{d-r} \leq d\right\},
$$

and let $\Upsilon=\Upsilon_{\nu}$ for an arbitrary $\nu$.

For each vertex $\nu \in V(\mathcal{T})$, define

$$
\operatorname{Mass}(\nu)=\prod_{\sigma \in[\varrho, \nu]} X_{\sigma}
$$

and for any set of vertices $A \subset V(\mathcal{T})$, let $\operatorname{Mass}(A)=\sum_{\nu \in A} \operatorname{Mass}(\nu)$.

Given non-negative integer $n$, consider level $n$ of $\mathcal{T}$, i.e. the set of vertices at distance $n$ from $\varrho$. Denote by $\mathcal{G}_{n, r}$ the collection of subsets of at most $r^{n}$ vertices at level $n$, with the additional property that they belong to the same $r$-ary subtree. 
The main result of this section is the following.

Lemma 2.1. Let $\lambda, \kappa$ be positive constants satisfying

$$
d \kappa^{\lambda} \mathbf{E}\left[(1-\Upsilon)^{\lambda}\right]<1
$$

Then eventually for large $n$,

$$
\max _{C \in \mathcal{G}_{n, r}} \operatorname{Mass}(C) \leq \kappa^{-n}
$$

For a given positive integer $n$ and a positive number $\kappa$, define the event

$$
\mathcal{C}_{n, \kappa}=\bigcap_{\nu:|\nu|=n}\left\{\prod_{\sigma \in\left[\varrho, \nu^{-}\right]}\left(1-\Upsilon_{\sigma}\right)^{-1} \geq \kappa^{n}\right\}
$$

and define the random variable

$$
N_{1}=N_{1}(\kappa)=\min \left\{n: \mathcal{C}_{j, \kappa} \text { holds for all } j \geq n\right\}
$$

We set $N_{1}=\infty$ if the set on the right-hand side is empty.

Lemma 2.2. If $\lambda, \kappa>0$ satisfy (2.2), then $N_{1}(\kappa)$ is a.s. finite.

Proof. By the first Borel-Cantelli lemma, it is enough to show that

$$
\sum_{n=1}^{\infty} d^{n} \mathbb{P}\left(\prod_{\sigma \in\left[\varrho, \nu^{-}\right]}\left(1-\Upsilon_{\sigma}\right)^{-1}<\kappa^{n}\right)<\infty
$$

where $\nu=\nu(n)$ denotes an arbitrary vertex with $|\nu|=n$. Since the $\Upsilon_{\sigma}$ are independent and $\lambda>0$, the above probability is by Markov's inequality

$$
\begin{aligned}
\mathbb{P}\left(\prod_{\sigma \in\left[\varrho, \nu^{-}\right]}\left(1-\Upsilon_{\sigma}\right)^{\lambda}>\kappa^{-\lambda n}\right) & \leq \mathbf{E}\left[\prod_{\sigma \in\left[\varrho, \nu^{-}\right]}\left(1-\Upsilon_{\sigma}\right)^{\lambda}\right] \kappa^{\lambda n} \\
& =\left(\mathbf{E}\left[(1-\Upsilon)^{\lambda}\right] \kappa^{\lambda}\right)^{n} .
\end{aligned}
$$

The inequality (2.4) now follows from (2.2).

For each vertex $\nu$, we define its adjusted mass, denoted by $\operatorname{AM}(\nu)$, as follows. For the root, $\operatorname{AM}(\varrho)=1$, and for all other vertices $\nu$,

$$
\operatorname{AM}(\nu)=\prod_{\sigma \in\left[\varrho, \nu^{-}\right]} X_{\sigma}\left(\frac{1}{1-\Upsilon_{\sigma}}\right)
$$

For any $A \subset V(\mathcal{T})$, let $\operatorname{AM}(A)=\sum_{\nu \in A} \operatorname{AM}(\nu)$. 
Lemma 2.3. For every positive integer $n$ and every $C \in \mathcal{G}_{n, r}$ we have $\operatorname{AM}(C) \leq 1$.

Proof. Let $C \in \mathcal{G}_{n, r}$. Define $\operatorname{Tree}_{r}(C)$ to be the $r$-ary subtree of $\mathcal{T}$ whose leaves are the vertices of $C$. For each vertex $\nu$ of $\operatorname{Tree}_{r}(C)$, denote its set of offspring in $\operatorname{Tree}_{r}(C)$ by $\nu_{\text {off }}$. Then by the definition of $\Upsilon$ in $(2.1)$,

$$
\operatorname{Mass}\left(\nu_{\mathrm{off}}\right) \leq\left(1-\Upsilon_{\nu}\right) \operatorname{Mass}(\nu)
$$

Thus $\operatorname{AM}\left(\nu_{\text {off }}\right) \leq \operatorname{AM}(\nu)$. Hence, for any $1 \leq k \leq n$, we have

$$
\sum_{\substack{\mu \in \operatorname{Tree}_{r}(C) \\|\mu|=k}} \operatorname{AM}(\mu) \leq \sum_{\substack{\nu \in \operatorname{Tree}_{r}(C) \\|\nu|=k-1}} \operatorname{AM}(\nu)
$$

Iterating this, we get

$$
\operatorname{AM}(C)=\sum_{\nu \in C} \operatorname{AM}(\nu) \leq \operatorname{AM}(\varrho)=1
$$

Proof of Lemma 2.1. Recall the definition of $N_{1}$ from (2.3). Lemma 2.2 implies that $N_{1}$ is a.s. finite. If $n \geq N_{1}$, then for any $C \in \mathcal{G}_{n, r}$ we have

$$
\operatorname{Mass}(C) \leq \kappa^{-n} \operatorname{AM}(C) \leq \kappa^{-n}
$$

where the last inequality is a consequence of Lemma 2.3 .

\section{$3 \quad$ Largest $r$-ary subtrees of random $d$-ary trees}

As the Beta and Dirichlet distributions play an important role in this paper, we recall their definitions.

Definition (Beta and Dirichlet distributions). Let $\Gamma(t)=\int_{0}^{\infty} x^{t-1} \mathrm{e}^{-x} \mathrm{~d} x$. For positive numbers $\alpha, \beta$, a random variable is said to be distributed as $\operatorname{Beta}(\alpha, \beta)$ if it has density

$$
\frac{\Gamma(\alpha+\beta)}{\Gamma(\alpha) \Gamma(\beta)} x^{\alpha-1}(1-x)^{\beta-1} \quad \text { for } x \in(0,1) .
$$

The multivariate generalization of the Beta distribution is called the Dirichlet distribution. Let $\alpha_{1}, \alpha_{2}, \ldots, \alpha_{n}$ be positive numbers. The Dirichlet $\left(\alpha_{1}, \alpha_{2}, \ldots \alpha_{n}\right)$ distribution has support on the set

$$
\left\{\left(x_{1}, x_{2}, \ldots, x_{n}\right): x_{i} \geq 0 \text { for } 1 \leq i \leq n, \text { and } \sum_{i=1}^{n} x_{i}=1\right\}
$$

and its density at point $\left(x_{1}, x_{2}, \ldots, x_{n}\right)$ equals

$$
\frac{\Gamma\left(\sum_{i=1}^{n} \alpha_{i}\right)}{\prod_{i=1}^{n} \Gamma\left(\alpha_{i}\right)} \prod_{j=1}^{n} x_{j}^{\alpha_{j}-1}
$$

Note that if the vector $\left(X_{1}, X_{2}, \ldots, X_{n}\right)$ is distributed as Dirichlet $\left(\alpha_{1}, \alpha_{2}, \ldots, \alpha_{n}\right)$, then the marginal distribution of $X_{i}$ is $\operatorname{Beta}\left(\alpha_{i}, \sum_{j \neq i} \alpha_{j}\right)$. 
Let $r$ and $d$ be fixed positive integers with $r<d$. Let $\left(B_{1}, B_{2}, \ldots, B_{d}\right)$ be a random vector distributed as Dirichlet $(1 /(d-1), 1 /(d-1), \ldots, 1 /(d-1))$, and define the random variable $\Upsilon$ as

$$
\Upsilon=\min \left\{B_{i_{1}}+B_{i_{2}} \ldots+B_{i_{d-r}}: 1 \leq i_{1}<i_{2}<\ldots<i_{d-r} \leq d\right\} .
$$

The main theorem of this section is the following.

Theorem 3.1. Let $r$ and $d$ be fixed positive integers with $r<d$, and let $S_{t}$ denote the size of the largest $r$-ary subtree of a random d-ary recursive tree at time $t$. Let $\tau, \kappa, \lambda$ be positive constants satisfying

$$
\begin{aligned}
e d \log \tau & <(d-1) \tau^{1 /(d-1)}, \\
d \kappa^{\lambda} \mathbf{E}\left[(1-\Upsilon)^{\lambda}\right] & <1,
\end{aligned}
$$

and let $n=\lfloor\log t / \log \tau\rfloor$. There exists a constant $K$ such that eventually (for large $t$ )

$$
S_{t} \leq K\left(r^{n}+t \kappa^{-n}\right) .
$$

Before proving this theorem, we show it implies Theorem 1.1.

Proof of Theorem 1.1. We show there exist positive constants $\tau, \kappa, \lambda$ satisfying (3.2), (3.3), and $\kappa>1$; then we would have $\tau>e^{d-1}>r$, and the theorem follows from Theorem 3.1 by choosing any $\delta \in(\max \{1-\log \kappa / \log \tau, \log r / \log \tau\}, 1)$. As (3.2) holds for all large enough $\tau$, it suffices to show there exist $\kappa>1$ and $\lambda>0$ satisfying (3.3). Since $\lim _{\varepsilon \rightarrow 0} \mathbb{P}(\Upsilon<\varepsilon)=$ 0 , we have $\mathbf{E}\left[(1-\Upsilon)^{\lambda}\right] \rightarrow 0$ as $\lambda \rightarrow \infty$. In particular, there exists $\lambda>0$ such that $\mathbf{E}\left[(1-\Upsilon)^{\lambda}\right]<1 / d$. Then, we can let

$$
\kappa=\left(d \mathbf{E}\left[(1-\Upsilon)^{\lambda}\right]\right)^{-1 /(2 \lambda)},
$$

which is strictly larger than 1 .

In the rest of this section, $\mathcal{T}$ denotes an infinite $d$-tree rooted at $\varrho$. We view the random recursive $d$-ary tree $\mathcal{T}_{t}$ as a subtree of $\mathcal{T}$. At each time step, we assign a weight to each vertex. For each $t$ and each vertex $\nu$ of $\mathcal{T}_{t}$, define $\aleph(\nu, t)$ to be the number of vertices $\mu$ of $\mathcal{T}_{t}$ such that $\nu \in[\varrho, \mu]$. This is the number of vertices in the "branch" of $\mathcal{T}_{t}$ containing $\nu$, including $\nu$. Set

$$
\operatorname{Weight}(\nu, t)=\frac{\aleph(\nu, t)-1}{d}
$$

if $\nu \in V\left(\mathcal{T}_{t}\right)$, and Weight $(\nu, t)=0$ if $\nu \notin V\left(\mathcal{T}_{t}\right)$. Note that this is the number of non-leaf vertices in this branch at time $t$.

Lemma 3.2. There exist random variables $\left\{B_{\nu}\right\}_{\nu \in V(\mathcal{T})}$, with $B_{\varrho}=1$, such that for any positive integer $t$ and any $\nu \in V(\mathcal{T})$, Weight $(\nu, t)$ is stochastically dominated by a Binomial $\left(t, \prod_{\sigma \in[\varrho, \nu]} B_{\sigma}\right)$ random variable. Moreover, the vectors $\left(B_{\nu 1}, B_{\nu 2}, \ldots, B_{\nu d}\right)$ are independent for $\nu \in V(\mathcal{T})$, and are distributed as Dirichlet $(1 /(d-1), 1 /(d-1), \ldots, 1 /(d-1))$. 
Proof. Consider a vertex $\nu \neq \varrho$ and a positive integer $t$ such that $\nu \in V\left(\mathcal{T}_{t}\right)$. Note that at time $t$, the number of leaves in the branch at $v$ is $(d-1)$ Weight $(\nu, t)+1$. Hence, given that at time $t+1$ the weight of $\nu^{-}$increases, the probability, conditional on the past, that the weight of $\nu$ increases at the same time, is equal to

$$
\frac{(d-1) \operatorname{Weight}(\nu, t)+1}{(d-1) \operatorname{Weight}\left(\nu^{-}, t\right)+1}
$$

Each time a weight increases, its increment is exactly 1. By identifying $\nu$ with one colour and its siblings with another colour, the evolution of the numerator of the above expression over time can be modelled using an Eggenberger-Pólya urn, with initial conditions $(1, d-1)$ and reinforcement equal to $d-1$. Moreover, the urns corresponding to distinct vertices are mutually independent.

The limiting distribution describing the Eggenberger-Pólya urn is well known, but we require bounds applying for all $t$. To this end, we can express the number of times a given colour is chosen by time $t$ in an Eggenberger-Pólya urn as a mixture of binomials with respect to a Beta distribution. See, for example, Pemantle [10, Lemma 1]. In this case, given the initial conditions $(1, d-1)$ and reinforcement $d-1$, the mixture is with respect to $\operatorname{Beta}(1 /(d-1), 1)$. This means that to each vertex $\nu$ we can assign a random variable $B_{\nu}$ distributed as $\operatorname{Beta}(1 /(d-1), 1)$, such that Weight $(\nu, t)$ conditional on $B_{\nu}$ is binomially distributed with parameters Weight $\left(\nu^{-}, t\right)-1$ and $B_{\nu}$. Set $B_{\varrho}=1$ and note that Weight $(\varrho, t)=t$. By induction, Weight $(\nu, t)$, conditional on $\left\{B_{\sigma}\right\}_{\sigma \in[\varrho, \nu]}$, is stochastically smaller than a $\operatorname{Binomial}\left(t, \prod_{\sigma \in[\varrho, \nu]} B_{\sigma}\right)$.

By the Eggenberger-Pólya urn representation we also infer that the joint distribution of $\left(B_{\nu 1}, B_{\nu 2}, \ldots, B_{\nu d}\right)$ is Dirichlet $(1 /(d-1), 1 /(d-1), \ldots, 1 /(d-1))$ for all $\nu$. See, for example, [10, Lemma 1].

Lemma 3.3. Let $B_{1}, \ldots, B_{n}$ be independent Beta $(1 /(d-1), 1)$ random variables. For all positive $\beta$ we have

$$
\mathbb{P}\left(\prod_{i=1}^{n} B_{i} \leq \beta^{n}\right) \leq\left(\frac{e \log (1 / \beta) \beta^{1 /(d-1)}}{d-1}\right)^{n}
$$

Proof. If $\beta \geq e^{1-d}$ then the right-hand side is at least 1 , so we may assume that $0<\beta<e^{1-d}$. We use Chernoff's technique. Let $\lambda \in(-1 /(d-1), 0)$ be a parameter which will be specified later. We have

$$
\mathbf{E}\left[B_{1}^{\lambda}\right]=\frac{\Gamma(d /(d-1))}{\Gamma(1 /(d-1)) \Gamma(1)} \int_{0}^{1} x^{\lambda} x^{-1+1 /(d-1)} \mathrm{d} x=\frac{1}{(d-1) \lambda+1} .
$$

Hence by Markov's inequality and since the $B_{i}$ are independent,

$$
\mathbb{P}\left(\prod_{i=1}^{n} B_{i} \leq \beta^{n}\right)=\mathbb{P}\left(\prod_{i=1}^{n} B_{i}^{\lambda} \geq \beta^{\lambda n}\right) \leq \prod_{i=1}^{n} \frac{\mathbf{E}\left[B_{1}^{\lambda}\right]}{\beta^{\lambda}}=\left(\frac{1}{\beta^{\lambda}((d-1) \lambda+1)}\right)^{n}
$$


To minimize the right-hand side we choose $\lambda=-1 /(d-1)-1 / \log \beta$, which is in the correct range since $0<\beta<e^{1-d}$. This gives

$$
\mathbb{P}\left(\prod_{i=1}^{n} B_{i} \leq \beta^{n}\right) \leq\left(\frac{e \log (1 / \beta) \beta^{1 /(d-1)}}{d-1}\right)^{n}
$$

We now prove Theorem 3.1.

Proof of Theorem 3.1. Let $\left\{B_{\nu}\right\}_{\nu \in V(\mathcal{T})}$ be as given by Lemma 3.2. Denote by $\mathcal{G}_{n, r}$ the collection of subsets of the vertices of $\mathcal{T}$ at level $n$, with the property that they belong to the same $r$-ary subtree. We apply Lemma 2.1 with Mass defined using $X_{\sigma}=B_{\sigma}$. Since (3.3) holds, we conclude that eventually for large $n$,

$$
\max _{C \in \mathcal{G}_{n, r}} \operatorname{Mass}(C) \leq \kappa^{-n}
$$

By Lemma 3.2, Weight $(\nu, t)$ is stochastically dominated by a $\operatorname{Binomial}(t, \operatorname{Mass}(\nu))$. Chernoff's bound for binomials implies (see, e.g., [8, Theorem 2.3(b)])

$$
\mathbb{P}(\operatorname{Weight}(\nu, t) \geq 2 t \operatorname{Mass}(\nu) \mid \operatorname{Mass}(\nu) \geq q) \leq \exp (-t q)
$$

for every positive $q$.

Since $\tau$ satisfies (3.2), there exists $\tau_{1}<\tau$ satisfying

$$
e d \log \tau_{1}<(d-1) \tau_{1}^{1 /(d-1)}
$$

Let $\beta=1 / \tau_{1}$. By Lemma 3.3, for any vertex $\nu$ at level $n$

$$
\mathbb{P}\left(\operatorname{Mass}(\nu)<\beta^{n}\right) \leq\left(\frac{e \log (1 / \beta) \beta^{1 /(d-1)}}{d-1}\right)^{n} .
$$

Note that (3.5) implies that the term in brackets is a constant smaller than $1 / d$.

We have

$$
\begin{aligned}
\mathbb{P}\left(\bigcup_{\mu:|\mu|=n}\{\operatorname{Weight}(\mu, t) \geq 2 t \operatorname{Mass}(\mu)\}\right) & \leq d^{n} \mathbb{P}(\operatorname{Weight}(\nu, t) \geq 2 t \operatorname{Mass}(\nu)) \\
& \leq d^{n} \mathbb{P}\left(\operatorname{Mass}(\nu)<\beta^{n}\right)+d^{n} \exp \left(-t \beta^{n}\right)
\end{aligned}
$$

The last expression is summable in $n$, as $t^{1 / n} \beta \geq \tau \beta$, and $\tau \beta$ is a constant larger than 1. By the first Borel-Cantelli lemma and (3.4), there exists a constant $K$ such that eventually for large $t$ we have $S_{t} \leq K\left(r^{n}+t \kappa^{-n}\right)$. 


\section{Longest paths in random Apollonian networks}

We define a tree $\mathcal{T}_{t}$, called the $\triangle$-tree of $\mathrm{RAN}_{t}$, as follows. There is a one to one correspondence between the triangles in $\mathrm{RAN}_{t}$ and the vertices of $\mathcal{T}_{t}$. For every triangle $\triangle$ in $\operatorname{RAN}_{t}$, we denote its corresponding vertex in $\mathcal{T}_{t}$ by $\mathbf{v}^{\triangle}$. To build $\mathcal{T}_{t}$, start with a single root vertex $\varrho$, which corresponds to the initial triangle of $R A N_{t}$. Wherever a triangle $\triangle$ is subdivided into triangles $\triangle_{1}, \triangle_{2}$, and $\triangle_{3}$, generate three offspring $\mathbf{v}^{\triangle_{1}}, \mathbf{v}^{\triangle_{2}}$, and $\mathbf{v}^{\triangle_{3}}$ for $\mathbf{v}^{\triangle}$, and extend the correspondence in the natural manner. Note that $\mathcal{T}_{t}$ is a random 3 -ary recursive tree as defined in the introduction and $\mathcal{T}_{t}$ has $3 t+1$ vertices and $2 t+1$ leaves. The leaves of $\mathcal{T}_{t}$ correspond to the bounded faces of $\mathrm{RAN}_{t}$. Let $\mathcal{T}$ denote an infinite 3 -ary tree rooted at $\varrho$. We view $\mathcal{T}_{t}$ as a subtree of $\mathcal{T}$. For each vertex $\nu \in V(\mathcal{T})$, the grand-offspring of $\nu$ are its descendants at level $|\nu|+2$. For a triangle $\triangle$ in $\operatorname{RAN}_{t}, I(\triangle)$ denotes the set of vertices of $\mathrm{RAN}_{t}$ that are strictly inside $\triangle$.

The following lemma was proved in [4, Lemma 3.1], and introduces the connection with the setup in Section 3.

Lemma 4.1. Let $\mathcal{T}_{t}$ be the $\triangle$-tree of $\mathrm{RAN}_{t}$. Let $\mathbf{v}^{\triangle}$ be a vertex of $\mathcal{T}_{t}$ with nine grandoffspring $\mathbf{v}^{\triangle_{1}}, \mathbf{v}^{\triangle_{2}}, \ldots, \mathbf{v}^{\triangle_{9}}$ in $V\left(\mathcal{T}_{t}\right)$. Then the vertex set of a path in $\mathrm{RAN}_{t}$ intersects at most eight of the $I\left(\triangle_{i}\right)$ 's.

We say that a finite subtree $\mathcal{J}$ of $\mathcal{T}$ is buono if each vertex of $\mathcal{J}$ has at most eight grand-offspring in $\mathcal{J}$.

Assume the four vectors $\left(A_{1}, A_{2}, A_{3}\right),\left(B_{1}, B_{2}, B_{3}\right),\left(B_{4}, B_{5}, B_{6}\right)$ and $\left(B_{7}, B_{8}, B_{9}\right)$ are i.i.d random vectors distributed as Dirichlet $(1 / 2,1 / 2,1 / 2)$. Define the random variable

$$
\Upsilon=\min \left\{A_{i} B_{j}: 1 \leq i \leq 3,1 \leq j \leq 9\right\}
$$

The main theorem of this section is the following.

Theorem 4.2. Let $\tau, \kappa, \lambda$ be positive constants satisfying

$$
\begin{aligned}
3 e \log \tau & <2 \sqrt{\tau} \\
9 \kappa^{\lambda} \mathbf{E}\left[(1-\Upsilon)^{\lambda}\right] & <1,
\end{aligned}
$$

and let $n$ be the largest even integer smaller than $\log t / \log \tau$. Then, there exists a constant $K$, such that eventually for large $t$, the largest buono subtree of $\mathcal{T}_{t}$ has at most $K\left(8^{n / 2}+t \kappa^{-n / 2}\right)$ vertices.

We show how this theorem implies Theorem 1.2.

Proof of Theorem 1.2. Following the proof of Theorem 1.1, we can find positive constants $\tau, \kappa, \lambda$ satisfying the conditions of Theorem 4.2, with $\kappa>1$ and $\tau>8$. Choose any $\delta \in$ $(\max \{1-\log (\kappa) /(2 \log \tau), \log (8) / 2 \log \tau\}, 1)$. 
Let $P$ be a path in RAN $_{t}$ and let $R(P)$ denote the set of vertices $\mathbf{v}^{\triangle}$ of $\mathcal{T}_{t}$ such that $I(\triangle)$ contains some vertex of $P$. By Lemma 4.1, $R(P)$ induces a buono subtree of $\mathcal{T}_{t}$. Hence, using Theorem 4.2 for the second inequality, eventually for large $t$ we have

$$
|V(P)| \leq 3+|R(P)| \leq 3+K\left(8^{n / 2}+t \kappa^{-n / 2}\right)<t^{\delta},
$$

as required.

The rest of this section is devoted to the proof of Theorem 4.2. For each time $t$ and vertex $\nu$ of $\mathcal{T}_{t}$, let $\aleph(\nu, t)$ denote the number of vertices $\mu$ of $\mathcal{T}_{t}$ such that $\nu \in[\varrho, \mu]$. Let

$$
\operatorname{Weight}(\nu, t)=\frac{\aleph(\nu, t)-1}{3}
$$

if $\nu \in V\left(\mathcal{T}_{t}\right)$, and $\operatorname{Weight}(\nu, t)=0$ if $\nu \notin V\left(\mathcal{T}_{t}\right)$.

By Lemma 3.2, there exist random variables $\left\{B_{\nu}\right\}_{\nu \in V(\mathcal{T})}$, such that for any positive integer $t$ and any $\nu \in V(\mathcal{T})$, Weight $(\nu, t)$ is stochastically dominated by a $\operatorname{Binomial}\left(t, \prod_{\sigma \in[\varrho, \nu]} B_{\sigma}\right)$. Moreover, $B_{\varrho}=1$ and for all $\nu \in V(\mathcal{T})$, the joint vectors $\left(B_{\nu 1}, B_{\nu 2}, B_{\nu 3}\right)$ are independent and distributed as Dirichlet $(1 / 2,1 / 2,1 / 2)$. Define $\operatorname{Mass}(\nu)=\prod_{\sigma \in[\varrho, \nu]} B_{\sigma}$.

Denote by $\mathcal{B}_{n}$ the collection of subsets of $V(\mathcal{T})$ at level $n$, with the property that they belong to the same buono subtree. Note that each element of $\mathcal{B}_{2 n}$ has at most $8^{n}$ vertices.

Lemma 4.3. Let $\lambda, \kappa$ be positive constants satisfying (4.3). Eventually, for large $n$,

$$
\max _{C \in \mathcal{B}_{2 n}} \operatorname{Mass}(C) \leq \kappa^{-n}
$$

Proof. Let $\mathcal{T}^{\prime}$ be an infinite rooted 9-ary tree obtained from $\mathcal{T}$ as follows. The vertices of $\mathcal{T}^{\prime}$ are the vertices of $\mathcal{T}$ at even levels. A vertex $\mu$ is an offspring of $\nu$ in $\mathcal{T}^{\prime}$ if $\mu$ is a grand-offspring of $\nu$ in $\mathcal{T}$. To each vertex $\mu$ assign the random variable $X_{\mu}=B_{\mu} B_{\mu^{-}}$. Buono subtrees of $\mathcal{T}$ are translated into 8-ary subtrees of $\mathcal{T}^{\prime}$. Using Lemma 2.1 concludes the proof.

We now prove Theorem 4.2. The proof is similar to that of Theorem 3.1.

Proof of Theorem 4.2. Recall that Weight $(\nu, t)$ is stochastically dominated by a Binomial $(\mathrm{t}, \operatorname{Mass}(\nu))$. Chernoff bound for binomials implies (see, e.g., [8, Theorem 2.3(b)])

$$
\mathbb{P}(\operatorname{Weight}(\nu, t) \geq 2 t \operatorname{Mass}(\nu) \mid \operatorname{Mass}(\nu) \geq q) \leq \exp (-t q)
$$

for every positive $q$.

Since $\tau$ satisfies (4.2), there exists $\tau_{1}<\tau$ satisfying

$$
3 e \log \tau_{1}<2 \sqrt{\tau_{1}} .
$$


Let $\beta=1 / \tau_{1}$. By Lemma 3.3, for any vertex $\nu$ at level $n$ we have

$$
\mathbb{P}\left(\operatorname{Mass}(\nu)<\beta^{n}\right) \leq\left(\frac{e \log (1 / \beta) \sqrt{\beta}}{2}\right)^{n} .
$$

Note that (4.4) implies that the term in brackets is a constant smaller than $1 / 3$.

We have

$$
\begin{aligned}
\mathbb{P}\left(\bigcup_{\mu:|\mu|=n}\{\operatorname{Weight}(\mu, t) \geq 2 t \operatorname{Mass}(\mu)\}\right) & \leq 3^{n} \mathbb{P}(\operatorname{Weight}(\nu, t) \geq 2 t \operatorname{Mass}(\nu)) \\
& \leq 3^{n} \mathbb{P}\left(\operatorname{Mass}(\nu)<\beta^{n}\right)+3^{n} \exp \left(-t \beta^{n}\right)
\end{aligned}
$$

The last expression is summable in $n$, as $t^{1 / n} \beta \geq \tau \beta$, and $\tau \beta$ is a constant larger than 1. By the first Borel-Cantelli lemma and Lemma 4.3, there exists a constant $K$ such that eventually for large $t$, the largest buono subtree of $\mathcal{T}_{t}$ has at most $K\left(8^{n / 2}+t \kappa^{-n / 2}\right)$ vertices.

\section{Appendix: Explicit bounds}

\subsection{Explicit bound for Theorem 1.1}

In this section we prove an explicit version of Theorem 1.1. For the proof we will need the following inequalities (see, e.g., Laforgia [7, Equations (2.2) and (2.3)]), valid for all $p, q>0$ and $0<\sigma<1<\iota<2$ :

$$
(p+\iota / 2)^{\iota-1}<\frac{\Gamma(p+\iota)}{\Gamma(p+1)}, \text { and } \quad \frac{\Gamma(q+\sigma)}{\Gamma(q+1)}<(q+\sigma / 2)^{\sigma-1} .
$$

Theorem 5.1. Let $r$ and $d$ be fixed positive integers with $r<d$, and let $S_{t}$ denote the size of the largest $r$-ary subtree of a random d-ary recursive tree at time $t$. Let

$$
\delta=1-\frac{d-r}{e d^{2 d} \log (11 d \log d)}
$$

Then eventually $S_{t}<t^{\delta}$.

Proof. In view of the proof of Theorem 1.1, it suffices to show there exist positive constants $\tau, \kappa, \lambda$ satisfying the following inequalities:

$$
\begin{aligned}
e d \log \tau & <(d-1) \tau^{1 /(d-1)}, \\
d \kappa^{\lambda} \mathbf{E}\left[(1-\Upsilon)^{\lambda}\right] & <1, \\
\log r / \log \tau<1-\log \kappa / \log \tau & <\delta
\end{aligned}
$$

where $\Upsilon$ is defined in $(3.1)$. 
Set

$$
\lambda=e d^{2 d-2} /(d-r), \quad \text { and } \quad \kappa=\exp \left(\frac{1}{\lambda(d-1)}\right) .
$$

Let $B$ be a $\operatorname{Beta}(1 /(d-1), 1)$ random variable.

We have

$$
\mathbb{P}(\Upsilon \leq \varepsilon) \leq d \mathbb{P}\left(B \leq \frac{\varepsilon}{d-r}\right)=d\left(\frac{\varepsilon}{d-r}\right)^{1 /(d-1)}
$$

Therefore,

$$
\begin{aligned}
\mathbf{E}\left[(1-\Upsilon)^{\lambda}\right] & =\int_{0}^{1} \mathbb{P}\left((1-\Upsilon)^{\lambda} \geq x\right) d x \\
& =\int_{0}^{1} \mathbb{P}\left(\Upsilon \leq 1-x^{1 / \lambda}\right) d x \\
& \leq \int_{0}^{1} d\left(\frac{1-x^{1 / \lambda}}{d-r}\right)^{1 /(d-1)} d x
\end{aligned}
$$

Using the change of variables $y=x^{1 / \lambda}$, we find

$$
\int_{0}^{1}\left(1-x^{1 / \lambda}\right)^{1 /(d-1)} d x=\frac{\Gamma(d /(d-1)) \Gamma(\lambda+1)}{\Gamma(\lambda+d /(d-1))}<\lambda^{-1 /(d-1)},
$$

where we have used the inequalities in (5.1) with $p=\lambda, \iota=d /(d-1), q=1$, and $\sigma=1 /(d-1)$. Thus,

$$
d \kappa^{\lambda} \mathbf{E}\left[(1-\Upsilon(r))^{\lambda}\right]<\frac{d^{2} \kappa^{\lambda}}{(\lambda(d-r))^{1 /(d-1)}}=1
$$

so (5.3) holds.

Let $\tau_{0}=(11 d \log d)^{d-1}$ and notice that e $d \log \tau_{0}<(d-1) \tau_{0}^{1 /(d-1)}$ since $d \geq 2$. Moreover,

$$
\frac{\log \kappa}{\log \tau_{0}}=\frac{d-r}{(d-1)^{2} e \log (11 d \log d) d^{2(d-1)}}>\frac{d-r}{e d^{2 d} \log (11 d \log d)} .
$$

Choose $\tau$ larger than $\tau_{0}$ such that

$$
\frac{\log \kappa}{\log \tau}>\frac{d-r}{e d^{2 d} \log (11 d \log d)} .
$$

We also have $\log r+\log \kappa<\log \tau$. Thus (5.2) and (5.4) hold as well, and the proof is complete.

\subsection{Explicit bound for Theorem 1.2}

In this section we provide an explicit value for $\delta$ in Theorem 1.2. For $\lambda>1$, define

$$
g(\lambda)=\frac{9 \lambda}{2(\lambda-1)^{3 / 2}}(\sqrt{\pi}+\sqrt{\pi} \log (\lambda-1) / 2+4 / 9) .
$$


Lemma 5.2. Let $\Upsilon$ be defined as (4.1), and let $\lambda>1$. Then $\mathbf{E}\left[(1-\Upsilon)^{\lambda}\right]<g(\lambda)$.

Proof. Let $B_{1}$ and $B_{2}$ be independent $\operatorname{Beta}(1 / 2,1)$ random variables. The density function of each of $B_{1}$ and $B_{2}$ is $1 /(2 \sqrt{x})$ if $x \in(0,1)$ and 0 elsewhere, hence we have

$$
\mathbb{P}\left(B_{1} B_{2} \leq \varepsilon\right)=\int_{0}^{1}\left(\int_{0}^{\min \{1, \varepsilon / x\}} \frac{1}{2 \sqrt{y}} \mathrm{~d} y\right) \frac{1}{2 \sqrt{x}} \mathrm{~d} x=\sqrt{\varepsilon}(1+\log (1 / \varepsilon) / 2) .
$$

Thus

$$
\begin{aligned}
\mathbf{E}\left[(1-\Upsilon)^{\lambda}\right] & =\int_{0}^{1} \mathbb{P}\left((1-\Upsilon)^{\lambda} \geq x\right) d x \\
& =\int_{0}^{1} \mathbb{P}\left(\Upsilon \leq 1-x^{1 / \lambda}\right) d x \\
& \leq 9 \int_{0}^{1} \mathbb{P}\left(B_{1} B_{2} \leq 1-x^{1 / \lambda}\right) d x \\
& =\frac{9}{2} \int_{0}^{1} \sqrt{1-x^{1 / \lambda}} \log \left(\frac{e^{2}}{1-x^{1 / \lambda}}\right) d x
\end{aligned}
$$

With $y=(\lambda-1)\left(1-x^{1 / \lambda}\right)$, we find

$$
\begin{aligned}
\mathbf{E}\left[(1-\Upsilon)^{\lambda}\right] & \leq \frac{9 \lambda}{2(\lambda-1)^{3 / 2}} \int_{0}^{\lambda-1} \sqrt{y} \log \left(\frac{e^{2}(\lambda-1)}{y}\right)\left(1-\frac{y}{\lambda-1}\right)^{\lambda-1} d y \\
& <\frac{9 \lambda}{2(\lambda-1)^{3 / 2}} \int_{0}^{\lambda-1} \sqrt{y} \log \left(\frac{e^{2}(\lambda-1)}{y}\right) e^{-y} d y .
\end{aligned}
$$

We have

$$
\int_{0}^{\infty} \sqrt{y} \log \left(e^{2}(\lambda-1)\right) e^{-y} d y=\log \left(e^{2}(\lambda-1)\right) \sqrt{\pi} / 2
$$

and

$$
\int_{0}^{\lambda-1} \sqrt{y} \log (1 / y) e^{-y} d y \leq \int_{0}^{1} \sqrt{y} \log (1 / y) e^{-y} d y \leq \int_{0}^{1} \sqrt{y} \log (1 / y) d y=4 / 9,
$$

concluding the proof.

Set $\lambda=10^{6}, \kappa=(9 g(\lambda))^{-1 / \lambda}, \tau=720$, and $\delta=1-4 \times 10^{-8}$. It is easily verified that $3 e \log \tau<2 \sqrt{\tau}$ and $\delta>\max \{1-\log (\kappa) / 2 \log \tau, \log (8) / 2 \log \tau\}$. Moreover, Lemma 5.2 implies that $9 \kappa^{\lambda} \mathbf{E}\left[(1-\Upsilon)^{\lambda}\right]<1$. As in the proof of Theorem 1.2, we get that $\mathcal{L}_{t}<t^{\delta}$ eventually.

\section{References}

[1] M. Albenque and J.-F. Marckert. Some families of increasing planar maps. Electron. J. Probab., 13:no. 56, 1624-1671, 2008. 
[2] C. Cooper and A. Frieze. Long paths in random Apollonian networks. arXiv, 1403.1472v1 [math.PR], 2014.

[3] M. Drmota. Random trees: An interplay between combinatorics and probability. SpringerWienNewYork, Vienna, 2009.

[4] E. Ebrahimzadeh, L. Farczadi, P. Gao, C. Sato, A. Mehrabian, N. Wormald, and J. Zung. On longest paths and diameter in random apollonian networks. Random Structures and Algorithms, 2014. published online.

[5] A. Frieze and C. E. Tsourakakis. On certain properties of random apollonian networks. In Anthony Bonato and Jeannette Janssen, editors, Algorithms and Models for the Web Graph, volume 7323 of Lecture Notes in Computer Science, pages 93-112. Springer Berlin Heidelberg, 2012.

[6] I. Kolossváry, J. Komjáty, and L. Vágó. Degrees and distances in random and evolving Apollonian networks. arXiv, arXiv:1310.3864v1 [math.PR], 2013.

[7] A. Laforgia. Further inequalities for the gamma function. Mathematics of Computation, 42(166):597-600, 1984.

[8] C. McDiarmid. Concentration. In Probabilistic methods for algorithmic discrete mathematics, volume 16 of Algorithms Combin., pages 195-248. Springer, Berlin, 1998.

[9] M. Mungan. Comment on "apollonian networks: Simultaneously scale-free, small world, euclidean, space filling, and with matching graphs". Phys. Rev. Lett., 106:029802, Jan 2011.

[10] R. Pemantle. Phase transitions for reinforced random walk and RWRE on trees. Annals of Probability, 16(3):1229-1241, 1988.

[11] Z. Zhang, F. Comellas, G. Fertin, and L. Rong. High-dimensional Apollonian networks. J. Phys. A, 39(8):1811-1818, 2006.

[12] T. Zhou, G. Yan, and B.-H. Wang. Maximal planar networks with large clustering coefficient and power-law degree distribution. Phys. Rev. E, 71:046141, Apr 2005. 